\title{
Diffuse large B-cell lymphoma with TEL/ETV6 translocation
}

\author{
Deborah W. Sevilla MD, Subhadra V. Nandula PhD, Adriana I. Colovai PhD, \\ Suzy Alexander MS, Vundavalli V. Murty PhD, Bachir Alobeid MD, Govind Bhagat MD*
}

\author{
Department of Pathology, Columbia University, New York, NY 10032, USA
}

Received 7 July 2008; revised 14 August 2008; accepted 19 August 2008

\author{
Keywords: \\ Translocation; \\ Diffuse Large \\ B-Cell Lymphoma; \\ TEL, ETV6; \\ Karyotype; \\ Cytogenetics
}

\begin{abstract}
Summary Cytogenetic abnormalities of chromosome $12 \mathrm{p}$ involving the TEL/ETV6 gene are observed in a variety of hematopoietic neoplasms including acute leukemias, myelodysplastic syndromes, and myeloproliferative disorders. Karyotypic aberrations, including rearrangements, deletions, and amplifications of chromosome 12p, have been documented in B-cell non-Hodgkin lymphoma; however, rearrangements targeting TEL have rarely been reported. Here we describe a diffuse large Bcell lymphoma that had a complex karyotype including $\mathrm{t}(9 ; 12)(\mathrm{q} 22 ; \mathrm{p} 13)$, which was confirmed by fluorescence in situ hybridization to represent rearrangement of TEL. Additional cytogenetic abnormalities included $\mathrm{t}(3 ; 14)(\mathrm{q} 27 ; \mathrm{q} 32)$ involving the variant, alternative breakpoint region of the BCL6 gene and del(6)(q13q23), resulting in the loss of 1 allele of BLIMP1. This case reiterates the importance of correlating morphologic and phenotypic findings with the results of cytogenetic analysis to avoid errors in diagnosing hematologic neoplasms and highlights the rare association of B-cell nonHodgkin lymphoma with aberrations of TEL.

(C) 2009 Elsevier Inc. All rights reserved.
\end{abstract}

\section{Introduction}

Translocations and deletions of chromosome $12 \mathrm{p} 13$ involving the $T E L$ gene occur in a variety of hematologic neoplasms, including acute myeloid and lymphoblastic leukemias, myelodysplastic syndromes (MDSs), and myeloproliferative disorders $[1,2]$. Aberrations of $12 p$ have also been reported in $15 \%$ to $22 \%$ of B-cell non-Hodgkin lymphomas (B-NHLs); however, the targeted genes are not yet known in most cases $[3,4]$. We report a unique case of diffuse large B-cell lymphoma (DLBCL), which occurred in a child with history of classic Hodgkin lymphoma and

* Corresponding author. Department of Pathology, College of Physicians and Surgeons, Columbia University, VC14-228, New York, NY 10032, USA.

E-mail address: gb96@columbia.edu (G. Bhagat). presented with multiple lytic bone lesions and pleural effusions at relapse. The lymphoma had a complex karyotype, which included translocation of $12 \mathrm{p} 13$ that was confirmed to involve the TEL gene; rearrangement involving the variant, alternative breakpoint region (ABR) of the $B C L 6$ gene; and 6q deletion.

\section{Methods}

The computed tomography-guided biopsy of a lytic lesion of the lumbar vertebral body (L5) and a cell block prepared from the pleural fluid were fixed in formalin $(10 \%$ neutral buffered). Paraffin-embedded sections of these specimens were stained with hematoxylin and eosin, and a cytospin preparation was stained with Wright-Giemsa for morphologic review. 


\subsection{Immunohistochemistry, in situ hybridization, and flow cytometry}

Immunohistochemical (IHC) stains were performed on the vertebral bone biopsy and pleural fluid cell block with the primary antibodies CD20, PAX5, BCL6, CD10, MUM1, BCL2, CD5, CD3, Ki-67, and p53 after heat-induced antigen retrieval; and Envision plus (DAKO, Carpinteria, $\mathrm{CA}$ ) and diaminobenzidine (DAB) were used for visualization. IHC staining for TEL was also performed on the bone biopsy using an antibody directed against ETV6 (clone HPA000264, rabbit polyclonal; Sigma, St Louis, MO). In situ hybridization for Epstein-Barr virus-encoded small RNAs (EBER) was performed using the supplied protocol (INFORM EBER; Ventana, Tucson, AZ).

Four-color flow cytometry of the pleural fluid was performed using FACSCalibur (Becton Dickinson, San Diego, CA) and the following primary antibodies: CD45, CD19, CD20, CD10, CD43, $\kappa$ and $\lambda$ light chains, CD3, CD16, and CD56 (Becton Dickinson). Data were analyzed using Cellquest software (Becton Dickinson).

\subsection{Cytogenetic analysis}

Giemsa banding was performed on metaphase preparations obtained after short-term (12 hours) culture of the pleural fluid using standard methods. Fluorescence in situ hybridization (FISH) analyses were performed on methanolacetic-fixed cells using IGH, BCL6(3q27), TEL/ETV6 (12p13), and $c-M Y C(8 \mathrm{q} 24)$ dual-color break-apart probes (VYSIS, Downers Grove, IL); a locus specific probe for BLIMP1(PRDM1)(6q21) [5]; and a CEP6 probe (VYSIS), according to standard protocols; and 200 to 300 cells were analyzed. Fluorescence signals were captured after counterstaining with 4'-6-diamidino-2-phenylindole (DAPI) using the Cytovision Imaging system attached to a Nikon Eclipse 600 microscope (Applied Imaging, Santa Clara, CA).

\subsection{Immunoglobulin heavy chain gene rearrangement}

Polymerase chain reaction analysis to detect rearrangement of the immunoglobulin heavy chain gene variable region was performed on a pleural fluid specimen using the Biomed-2 primers (IVS Gene Clonality Assay Kit; InVivoScribe Technologies, San Diego, CA).

\subsection{Case report}

A 22-year-old woman was diagnosed with stage 4B classic Hodgkin lymphoma, nodular sclerosis type, 8 years

Fig. 1 A, Cytospin of pleural fluid showing large pleomorphic lymphocytes (Wright-Giemsa, 400×). B, Vertebral body biopsy showing an infiltrate of DLBCL (hematoxylin and eosin, 400×), with (C) intense nuclear staining for TEL (ETV6, 400×).
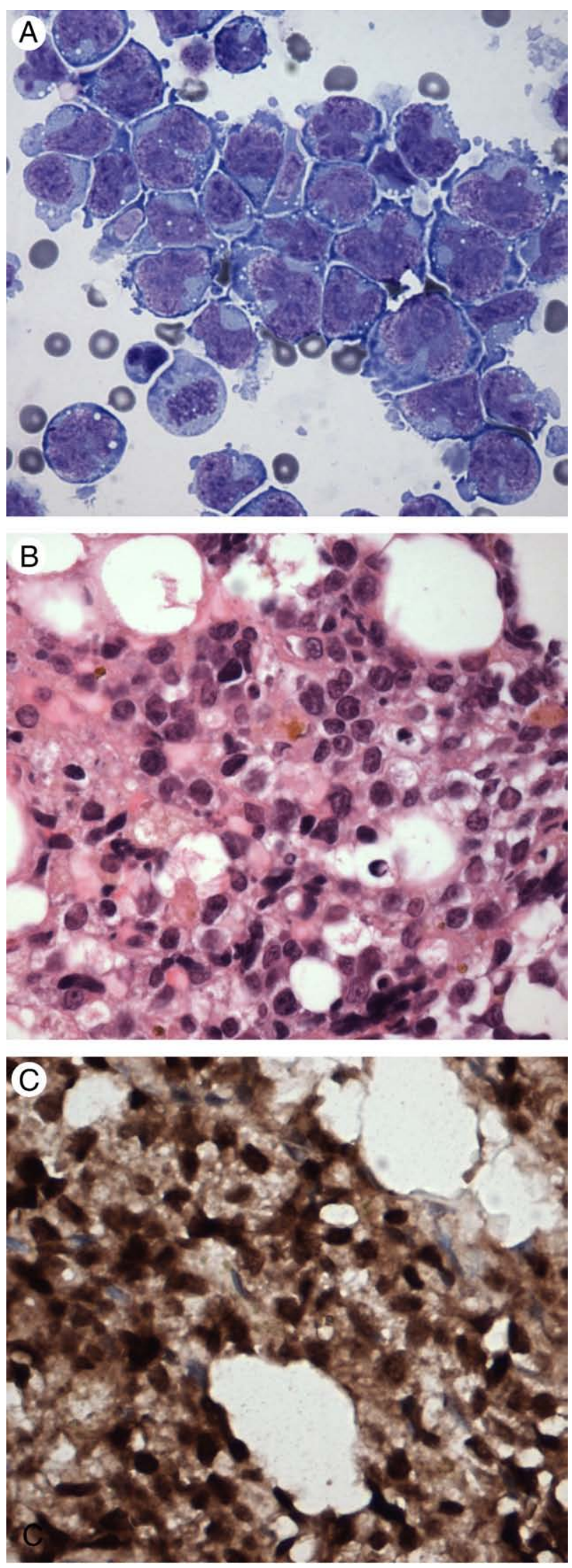
before her current presentation. She was refractory to a variety of chemotherapeutic regimens, including 8 cycles of COPP-ABVD (cyclophosphamide, prednisone, procarbazine, vincristine, bleomycin, dacarbazine, doxorubicin, vinblastine) followed by 2 cycles of ICE (carboplatin, etoposide, ifosfamide), high-dose $\mathrm{BCNU}$ (carmustine), etoposide, and autologous stem cell rescue, experiencing multiple relapses. Remission was eventually achieved after 10 cycles

A

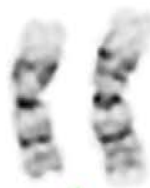

1

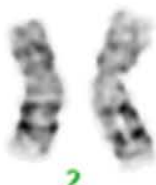

2

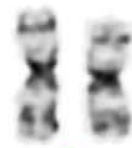

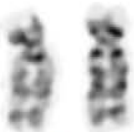

4

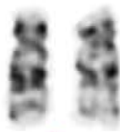

5

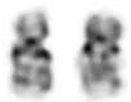

10

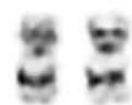

11
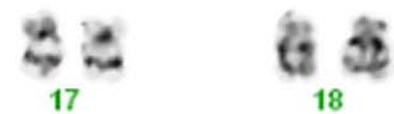

13
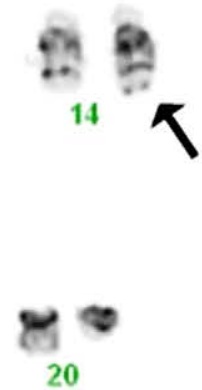

19

20
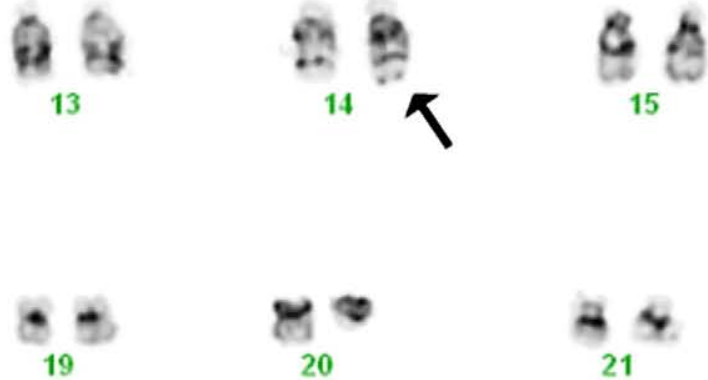

21

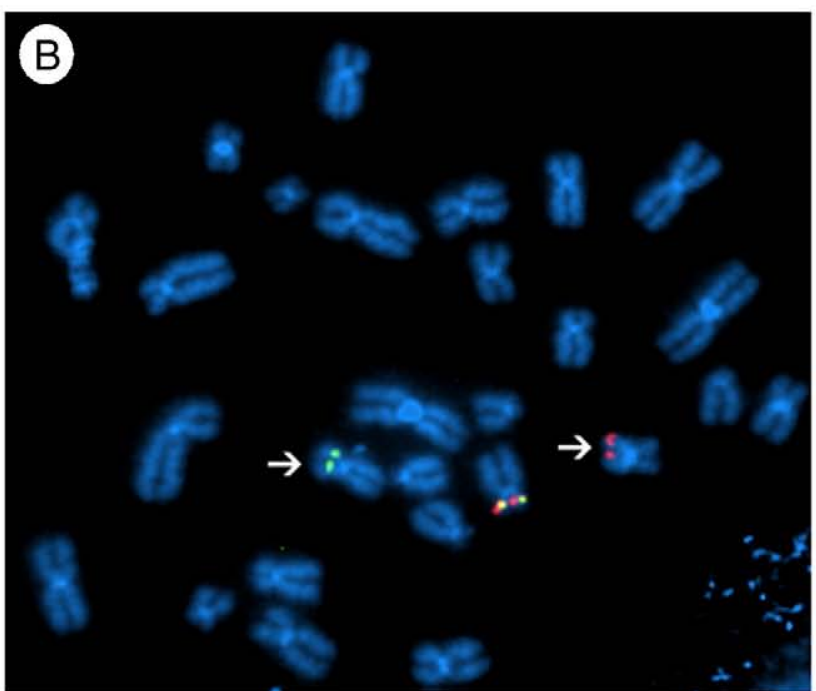

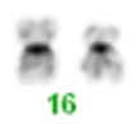
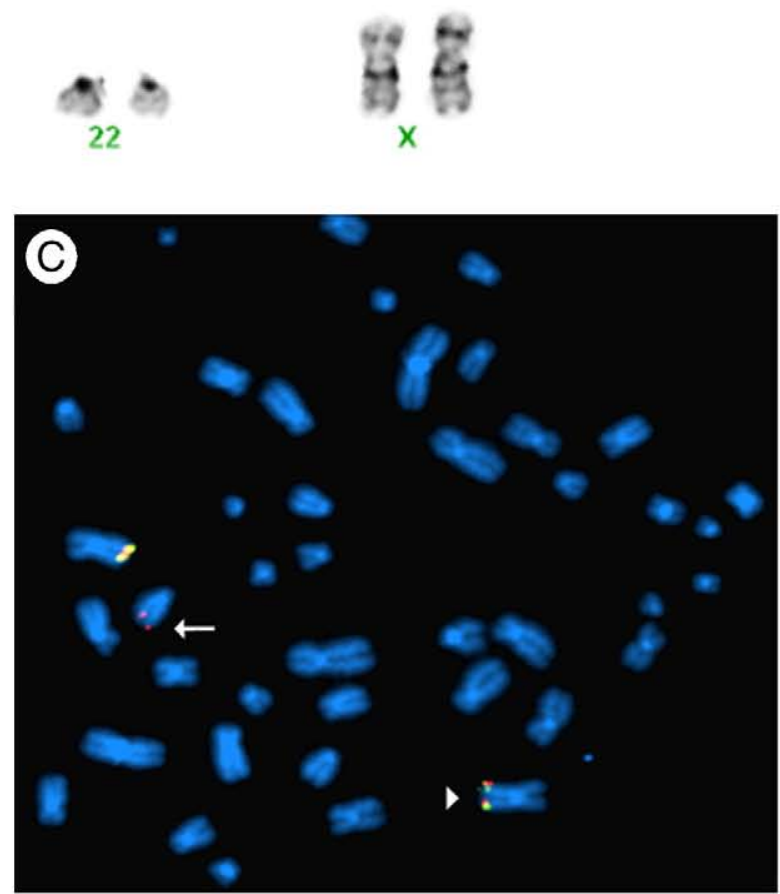

Fig. 2 A, G-band karyotype obtained from culture of pleural fluid. Arrows indicate $\mathrm{t}(9 ; 12), \mathrm{t}(3 ; 14)$, and del(6q)(q13q23). B, FISH with TEL break-apart probes shows 1 green (split) signal on the short arm of the abnormal chromosome 12 and 1 red (split) signal on the long arm of the abnormal chromosome 9 consistent with TEL translocation (arrows). A single yellow (fusion) signal is present on the normal chromosome 12. C, FISH with BCL6 break-apart probe shows 1 small red signal (arrow) translocated to the long arm of chromosome 14 and 1 green-yellow signal (unequal split, arrowhead) on the long arm of chromosome 3 consistent with ABR translocation; 1 yellow (fusion) signal (normal allele) on the nonrearranged chromosome 3 is also present. 
of gemcitabine and vinorelbine with mantle field (chest, neck, and axilla) radiation. Three years before presentation at our institute, she developed pancytopenia; and a bone marrow biopsy showed an infiltrate of DLBCL. She was then treated with CHOP (cyclophosphamide, doxorubicin, prednisone, vincristine) in conjunction with vincristine, cyclophosphamide, prednisone, doxorubicin, and rituximab for 6 months, again relapsing with bone marrow and central nervous system involvement. She was started on the COG 5961 (group C) consolidation-phase regimen with high-dose Ara-C and etoposide with rituximab, intrathecal triple therapy, and conditioning with fludarabine and busulfan in preparation for a bone marrow transplant. She then underwent a reducedintensity stem cell transplant from her mother 4 months before current presentation. Her most recent presentation was due to development of a large left pleural effusion, and positron emission tomographic scans showed increased uptake in the sternal manubrium and the L5 vertebral body.

Cytologic examination of the pleural fluid was notable for increased numbers of large atypical lymphocytes that had large round to ovoid nuclei, with occasional cells showing irregular nuclear contours, fine chromatin, multiple small nucleoli, and moderate to abundant basophilic cytoplasm (Fig. 1A). Biopsy of the vertebral lytic bone lesion revealed an infiltrate of large lymphocytes with similar morphologic features (Fig. 1B). Flow cytometry of the pleural fluid showed a population of $\lambda$-light chain restricted medium to large B-cells exhibiting intermediate to high forward scatter; bright CD45, CD19, CD20, and CD43 expression; and dim, partial CD10 expression. IHC staining of the vertebral bone biopsies and pleural fluid cell block demonstrated the following phenotypes of the neoplastic cells: CD20+, PAX5+, BCL6+, MUM1+, BCL2+, and CD43+. The lymphocytes also showed intense nuclear staining for TEL protein (Fig. 1C). The proliferation index, as assessed with a $\mathrm{Ki}-67$ stain, was markedly elevated (90\%). Aberrant nuclear expression of p53 was seen in most cells $(80 \%)$. Result of in situ hybridization for Epstein-Barr virus (EBER) was negative.

Cytogenetic analysis performed on the pleural fluid sample showed a complex karyotype (Table 1, Fig. 2A). FISH using the ETV6 (TEL)(12p13) break-apart probe confirmed rearrangement of TEL (split red and green signals, Fig. 2B). A probe for BLIMP1(PRDM1)(6q21) indicated loss of 1 copy of BLIMP1. IGH and BCL6 break-apart probes confirmed $\mathrm{t}(3 ; 14)$. However, the signal pattern (unequal split of signals) was consistent with a break $5^{\prime}$ to the BCL6 gene, involving the ABR (Fig. 2C). No MYC translocation was detected by FISH analysis.

A clonal product was detected by polymerase chain reaction for immunoglobulin heavy chain rearrangement in the pleural fluid sample. Together, these findings were diagnostic of a DLBCL involving the lumbar spine and pleural fluid.

The patient was admitted for management of her pleural effusion and consideration of another bone marrow trans- plant. Her hospital course was complicated by sepsis, renal failure, and disseminated intravascular coagulation; and she died shortly after admission.

\section{Discussion}

We describe a case of DLBCL with a complex karyotype and rearrangement of the TEL gene. To the best of our knowledge, this is only the fourth reported case of B-NHL with TEL rearrangement (Table 1) [6-8]. The precise morphologic, phenotypic, and clinical details of 2 cases are unknown because they were reported only as B-NHL that occurred in a 4-year-old girl [7] and a 30-year-old man [6]. The third reported case [8] was of a marginal zone lymphoma occurring in a 70-year-old man with involvement of the bone marrow, blood, lymph node, and spleen. That patient was alive 10 months after treatment with chlorambucil.

Rearrangements involving the TEL gene (also known as the E26 transforming specific [ETS] translocation variant gene 6 ; ETV6) are frequently detected in a variety of hematopoietic neoplasms, specifically acute leukemias, MDSs, and myeloproliferative disorders [1,2]. However, since the initial characterization of this gene and identification of TEL rearrangements and deletions over a decade ago, the function of TEL remains unclear. Structural studies have demonstrated that TEL contains both a $3^{\prime}$ ETS DNA binding domain, which also mediates protein-protein interactions, and a $5^{\prime}$ helix-loop-helix domain involved in homo-and heterodimerization of transcription factors and signal transducing proteins [9]. TEL is involved in chromosomal translocations with a variety of partners forming chimeric fusion transcripts (eg, TEL-PDGFRB, TEL-ABL, TEL$A M L 1$ ), and the function of TEL is thought to differ depending on the fusion partner. Bohlander [1] subdivided the fusion partners into different functional groups, for example, tyrosine kinases, transcription factors, as well as "unproductive fusions," in an attempt to further elucidate the role of TEL in hematologic disorders. Within the first category, TEL partnering with $J A K 2$, a tyrosine kinase fusion protein, leads to constitutive activation of the protein tyrosine kinase domain [10], whereas in cases of TEL-AML1 rearrangement, it is hypothesized that the chimeric fusion protein causes constitutive repression of $A M L 1$ leading to deregulation of $A M L 1$ target genes [11]. Finally, in addition to rearrangements involving TEL and formation of fusion genes or protooncogene activation, recurrent loss of genetic material from $12 \mathrm{p} 13$, the region containing the TEL gene, is also frequently described, suggesting possible function as a tumor suppressor. In many hematologic disorders with TEL rearrangement, deletion of the nonrearranged allele of $T E L$ occurs in $60 \%$ to $75 \%$ of cases [3,7]. Such a concomitant deletion was described in 2 of the previous B-NHLs with TEL rearrangement (cases 3 and 4), but was not detected in our case (Table 1). Of note, allelic loss of a microsatellite 
Table 1 B-NHLs with TEL rearrangements

\begin{tabular}{|c|c|c|c|c|}
\hline Case & Age/Sex & G-band karyotype & Diagnosis & Ref \\
\hline 1 & $22 / \mathrm{F}$ & $\begin{array}{l}\text { 46, XX,t(3;14)(q27;q32),del(6)(q13q23),t(9;12)(q22;p13), } \\
\operatorname{del}(20)(\mathrm{q} 11.2)[11] / 46, \operatorname{idem}, \mathrm{t}(1 ; 16)(\mathrm{q} 44 ; \mathrm{p} 13.1)[9]\end{array}$ & DLBCL & Current case \\
\hline 2 & $30 / \mathrm{M}$ & $46, X Y, t(2 ; 12)(q 31 ; p 13), \operatorname{del}(11)(q 21)$ & B-NHL & [6] \\
\hline 3 & $4 / \mathrm{F}$ & $\begin{array}{l}\text { 47,X,der(X)del(X)(q2?6;q2?8)t(X;12)(q2?6;p13);del(12)(p11p13), } \\
\operatorname{der}(12) \mathbf{t}(\mathbf{X} ; 12)(\mathbf{q} 2 \mathbf{6} ; \mathbf{p 1 3}),+21[13] / 47, \text { idem,add(13)(q32)[14] }\end{array}$ & B-NHL & [7] \\
\hline 4 & $70 / \mathrm{M}$ & $\begin{array}{l}\text { 48, XY,der(1)t(1;17)(p34;q24)add(1)(q21), der(5)t(5;12;?)(p11;p11p13;?), } \\
\operatorname{der}(7) \mathrm{t}(1 ; 7)(\mathrm{q} 21 ; \mathrm{q} 31), \operatorname{der}(12) \operatorname{del}(12)(\mathrm{p} 13 \mathrm{p} 13) \mathrm{t}(12 ; 14)(\mathrm{p} 11 ; \mathrm{q} 32), \\
\operatorname{dic}(12 ; 17)(\mathrm{p} 11 ; \mathrm{p} 11) \operatorname{del}(12)(\mathrm{p} 13 \mathrm{p} 13), \operatorname{del}(14)(\mathrm{q} 32), \operatorname{der}(14) \mathrm{t}(12 ; 14)(\mathrm{p} 11 ; \mathrm{q} 32), \\
\operatorname{der}(17) \mathrm{t}(1 ; 17)(\mathrm{p} 34 ; \mathrm{q} 24), \operatorname{der}(\mathbf{1 7}) \mathrm{t}(\mathbf{1 2} ; \mathbf{1 7})(\mathbf{p 1 3} ; \mathbf{1 1}),-19,-20,-2,+5 \mathrm{mar}\end{array}$ & MZBCL & {$[8]$} \\
\hline
\end{tabular}

Abbreviation: MZBCL, marginal zone B-cell lymphoma.

within the TEL gene has been documented on transformation of follicular lymphoma [4], further supporting a tumorsuppressor function in certain contexts. It would be of interest to see if other mechanisms leading to loss of function of TEL (eg, mutations) play a role in progression of B-NHL. Our case demonstrated TEL rearrangement with the locus $9 \mathrm{q} 22$, a region that has previously not been described in BNHL. However, rearrangement involving this locus has been reported in a case of MDS [12]. In the latter, the fusion partner on $9 \mathrm{q} 22$ was identified as tyrosine kinase gene $S y k$; and the TEL-Syk fusion was shown to lead to interleukin-3independent growth of $\mathrm{Ba} 3$ cells [12].

The time of acquisition of TEL rearrangement in our case is not clear because cytogenetic analysis was not performed at the time of primary presentation of the DLBCL. It is possible that the translocation of TEL occurred later during clonal evolution. The patient had received multiple chemotherapeutic regimens for both Hodgkin lymphoma and DLBCL; and thus, a role, if any, of these agents in inducing rearrangement of TEL cannot be excluded. A role of TEL in mediating resistance to chemotherapy is also unclear. Of note, 3 of the 4 B-NHLs with TEL translocations (including our case) had a complex karyotype (Table 1). Thus, as has been previously hypothesized, TEL rearrangement in these cases may represent a class II mutation, blocking differentiation and/or interfering with apoptosis, and, although required, is not sufficient for development of malignancy [13].

In the absence of fresh or frozen material, we could not analyze the messenger RNA expression level of TEL. However, intense nuclear staining for TEL was observed in the neoplastic lymphocytes by IHC analysis. This finding is suggestive of TEL overexpression because normal lymph node and tonsillar B-cells did not show any staining for TEL. It is unclear at present whether mechanisms other than translocations can also lead to deregulated expression of TEL in certain B-NHLs because a survey of TEL messenger RNA or protein expression in B-NHLs has not been performed. It should be mentioned that although we observed overexpression of $T E L$, the functional status of $T E L$ protein in our case is unknown.

Other genes on chromosome $12 p$ have been previously implicated in B-NHL. These include p27 $7^{\mathrm{Kip} 1}$, a cyclin- dependent kinase inhibitor located centromeric to TEL, which is expressed in lower levels in high-grade B-NHL, including DLBCL and mantle cell lymphoma [14-16]. The cyclin-D2 gene (CCND2) is located telomeric to TEL. Cyclin D2 overexpression via amplification or rearrangement with immunoglobulin heavy or light chains has been described in mantle cell lymphoma and more rarely in other B-NHLs, including Burkitt lymphoma and chronic lymphocytic leukemia (at transformation) [3,17-19]. Rearrangements involving $12 p$ are often accompanied by deletions in the adjacent regions. Because the size of the deletions is often large, at times encompassing the loci of both TEL and $\mathrm{p} 27^{\mathrm{Kip} 1}$, an impact of a loss of individual genes, or a combination of both, on disease inception or evolution of previously reported cases is unknown [3,6,7]. The findings of G-band karyotype and FISH ruled out structural aberrations of $\mathrm{p} 27^{\mathrm{Kip} 1}$ and $C C N D 2$ in the current case.

In addition to rearrangement of TEL, our DLBCL demonstrated a translocation involving the $\mathrm{ABR}$ of the $B C L 6$ gene. This breakpoint is located between 245 and 285 kilobases $5^{\prime}$ to $B C L 6$, and translocations involving this region are more commonly seen in follicular lymphomas; however, they are also detected in approximately $12 \%$ of DLBCLs with $3 \mathrm{q} 27$ translocation and $4 \%$ of DLBCLs overall [20]. Karyotype analysis also showed deletion of 6q, and FISH confirmed loss of 1 allele of the BLIMP1 gene. $B L I M P 1$ is a transcriptional repressor that is required for terminal B-cell differentiation. Alterations or loss of BLIMP1 has been described in $25 \%$ of activated B-cell like DLBCLs and are considered uncommon in germinal center B-cell like DLBCLs [5]. Because BCL6 translocations and $B L I M P 1$ deletions are considered mutually exclusive events [5], it has been hypothesized that alterations of genes leading to a block in plasma cell differentiation might be redundant in the same neoplasm. The significance of both these lesions occurring in our case is not known. It is possible that the deletion of BLIMP1 may represent a nonspecific or "bystander" deletion due to the large interstitial deletion of $6 \mathrm{q}$.

Lastly, but importantly, the rarity of TEL rearrangements in the setting of B-NHL, as described in our case, highlights a potential diagnostic pitfall if results of cytogenetic analysis 
are interpreted in the absence of morphologic and phenotypic data. This is especially pertinent for pediatric hematopoietic malignancies because rearrangements of TEL are seen in approximately $25 \%$ of cases of B-cell acute lymphoblastic leukemia [1].

\section{References}

[1] Bohlander SK. ETV6: a versatile player in leukemogenesis. Semin Cancer Biol 2005; 15:162-74.

[2] Mitelman F. Catalog of chromosome aberrations in cancer. New York: Liss; 1991

[3] Hoglund M, Johansson B, Pedersen-Bjergaard J, et al. Molecular characterization of $12 p$ abnormalities in hematologic malignancies: deletion of KIP1, rearrangement of TEL, and amplification of CCND2. Blood 1996;87:324-30.

[4] Takeuchi S, de Vos S, Takeuchi N, et al. Allelic loss during progression of follicular lymphoma. Leuk Res 2004;28:567-9.

[5] Pasqualucci L, Compagno M, Houldsworth J, et al. Inactivation of the PRDM1/BLIMP1 gene in diffuse large B cell lymphoma. J Exp Med 2006;203:311-7.

[6] Kobayashi H, Montgomery KT, Bohlander SK, et al. Fluorescence in situ hybridization mapping of translocations and deletions involving the short arm of human chromosome 12 in malignant hematologic diseases. Blood 1994;84:3473-82.

[7] Sato Y, Suto Y, Pietenpol J, et al. TEL and KIP1 define the smallest region of deletions on $12 \mathrm{p} 13$ in hematopoietic malignancies. Blood 1995;86:1525-33.

[8] Wlodarska I, La Starza R, Baens M, et al. Fluorescence in situ hybridization characterization of new translocations involving TEL (ETV6) in a wide spectrum of hematologic malignancies. Blood 1998; 91:1399-406.

[9] Grimshaw SJ, Mott HR, Stott KM, et al. Structure of the sterile alpha motif (SAM) domain of the Saccharomyces cerevisiae mitogenactivated protein kinase pathway-modulating protein STE50 and analysis of its interaction with the STE11 SAM. J Biol Chem 2004; 279:2192-201.

[10] Schwaller J, Frantsve J, Aster J, et al. Transformation of hematopoietic cell lines to growth-factor independence and induction of a fatal myelo-and lymphoproliferative disease in mice by retrovirally transduced TEL/JAK2 fusion genes. Embo J 1998;17:5321-33.

[11] Zelent A, Greaves M, Enver T. Role of the TEL-AML1 fusion gene in the molecular pathogenesis of childhood acute lymphoblastic leukaemia. Oncogene 2004;23:4275-83.

[12] Kuno Y, Abe A, Emi N, et al. Constitutive kinase activation of the TEL-Syk fusion gene in myelodysplastic syndrome with $\mathrm{t}(9 ; 12)(\mathrm{q} 22$; p12). Blood 2001;97:1050-5.

[13] Speck NA, Gilliland DG. Core-binding factors in haematopoiesis and leukaemia. Nat Rev Cancer 2002;2:502-13.

[14] Chiarle R, Budel LM, Skolnik J, et al. Increased proteasome degradation of cyclin-dependent kinase inhibitor p27 is associated with a decreased overall survival in mantle cell lymphoma. Blood 2000;95:619-26.

[15] Lloyd RV, Erickson LA, Jin L, et al. p27kip1: a multifunctional cyclindependent kinase inhibitor with prognostic significance in human cancers. Am J Pathol 1999;154:313-23.

[16] Quintanilla-Martinez L, Davies-Hill T, Fend F, et al. Sequestration of p27Kip1 protein by cyclin D1 in typical and blastic variants of mantle cell lymphoma (MCL): implications for pathogenesis. Blood 2003; 101:3181-7.

[17] Gesk S, Klapper W, Martin-Subero JI, et al. A chromosomal translocation in cyclin D1-negative/cyclin D2-positive mantle cell lymphoma fuses the CCND2 gene to the IGK locus. Blood 2006;108: 1109-10.

[18] Herens C, Lambert F, Quintanilla-Martinez L, et al. Cyclin D1negative mantle cell lymphoma with cryptic $\mathrm{t}(12 ; 14)(\mathrm{p} 13 ; \mathrm{q} 32)$ and cyclin D2 overexpression. Blood 2008;111:1745-6.

[19] Qian L, Gong J, Liu J, et al. Cyclin D2 promoter disrupted by $\mathrm{t}(12 ; 22)$ (p13;q11.2) during transformation of chronic lymphocytic leukaemia to non-Hodgkin's lymphoma. Br J Haematol 1999;106:477-85.

[20] Butler MP, Iida S, Capello D, et al. Alternative translocation breakpoint cluster region $5^{\prime}$ to BCL-6 in B-cell non-Hodgkin's lymphoma. Cancer Res 2002;62:4089-94. 